\title{
Growth, Morphology and Molecular Orientation of Controlled Indigo thin films on Silica Surfaces
}

Arianna Rivalta, ${ }^{,[a]}$ Cristiano Albonetti, ${ }^{,[b]}$ Davide Biancone, ${ }^{[b]}$ Michele Della Ciana,${ }^{[b, c]}$ Simone d'Agostino, ${ }^{[d]}$ Laure Biniek, ${ }^{[\mathrm{e}]}$ Martin Brinkmann ${ }^{[\mathrm{e}]}$ Andrea Giunchi, ${ }^{[\mathrm{a}]}$ Tommaso Salzillo, ${ }^{[\mathrm{a}]}{ }^{\dagger}$ Aldo Brillante, ${ }^{[\mathrm{a}]}$ Raffaele Guido Della Valle, ${ }^{[\mathrm{a}]}$ and Elisabetta Venuti ${ }^{*}[\mathrm{a}]$

[a] Department of Industrial Chemistry "Toso Montanari” and INSTM-UdR Bologna, University of Bologna, Viale del Risorgimento, 4 - 40136 Bologna (Italy)

${ }^{[b]}$ Consiglio Nazionale delle Ricerche - Istituto per lo Studio dei Materiali Nanostrutturati (CNR-ISMN) via P. Gobetti 101, 40129 Bologna, Italy

${ }^{[c]}$ Consiglio Nazionale delle Ricerche - Istituto per la Microelettronica e i Microsistemi (CNR-IMM) via P. Gobetti 101, 40129 Bologna, Italy

${ }^{\text {[d] }}$ Chemistry Department “Giacomo Ciamician', University of Bologna, Via F. Selmi 2, 40126 Bologna, Italy

[e] Université de Strasbourg, CNRS, Institut Charles Sadron, UPR 22, F-67000 Strasbourg, France

$\uparrow$ Present address: Department of Materials and Interfaces, Weizmann Institute of Science, P.O. Box 26, Rehovot 76100, Israel

\section{Abstract}

Thin films of the natural semiconductor indigo have been grown on $\mathrm{Si}_{/} \mathrm{SiO}_{2}$ substrates by physical vapour deposition under ultra-high vacuum conditions, monitoring the influence of the deposition rate on structure, morphology and thickness. This was done to allow well-defined films to be made in a controlled way, suitable for the manufacture of organic devices and the investigation of charge transport phenomena. The films have been characterized by X-ray diffraction, atomic force microscopy, microRaman spectroscopy and transmission electron microscopy, finding that the growth at the solid interface of polymorph $\mathrm{B}$, the only phase present in the films, is governed by the formation of strong multiple hydrogen-bonds between neighbouring molecules, which play a predominant role in the interface organization of the molecular assemblies.

\section{Introduction}

In modern science and technology of nanomaterials, interest in biological and bio-inspired systems is motivated by their potential for biomedical integration and sustainable development. ${ }^{1}$ At the same time, research in the field of organic semiconductor-based devices has grown extensively ${ }^{2}$ and has led to mature OLED technology as well as to highly promising applications in Organic Photovoltaics $(\mathrm{OPV})^{3-5}$ and Organic Field Effect Transistors (OFETs). ${ }^{6,7}$ The wide range of potential uses and the commercial success of organic semiconductors (OSCs), combined with the increasingly urgent issue of eco-compatibility, has stimulated the search for non-toxic, biodegradable, and biocompatible 
compounds also in this field. Thus, bio-inspired OSCs, which hold the promise of being environmentally friendly and of special relevance for bio-applications, have already been tested in organic thin film transistors (OTFTs), ${ }^{2,8-10}$ demonstrating interesting properties.

A much sought-after property in these systems is a balanced ambipolar charge transport, which is essential for the development of integrated microelectronic circuits and optoelectronic devices. Widespread applications, unfortunately, are currently hampered by the narrow choice of available compounds. ${ }^{11}$

The vat dye indigo [2,2'-Bis(2,3-dihydro-3-oxoindolyliden)] (Figure 1) holds the characteristics of both being an intrinsically ambipolar organic semiconductor and having a natural origin, even though its mobilities have not been measured yet in single crystal. ${ }^{11}$

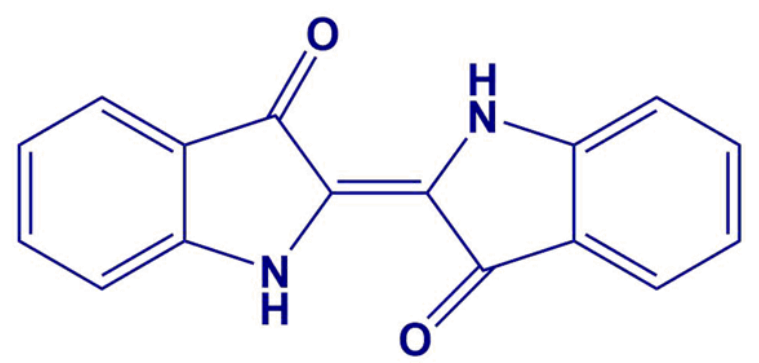

Figure 1: Molecular structure of indigo [2,2'-bis(2,3-dihydro-3-oxoindolyliden)].

Indigo has been shown to be a promising biocompatible semiconductor in OTFTs, ${ }^{2,11-15}$ with a bandgap of $1.7 \mathrm{eV}$, well-balanced electron and hole mobilities of $1 \times 10^{-2} \mathrm{~cm}^{2} \mathrm{~V}^{-1} \mathrm{~s}^{-1}$ and good stability of the hole transport against degradation in air. ${ }^{11,15}$ However, its OTFT mobility is still remarkably lower than that of best performing systems, ${ }^{16-18}$ which include rubrene, pentacene, substituted pentacenes, ${ }^{19,20}$ perylene diimide ${ }^{21-23}$ and thienoacene derivatives, ${ }^{24}$ and for actual applications it must be increased. For most of the above OSCs, with pentacene taken as a typical case ${ }^{25,26}$ growth parameters and electrical performances of devices have been investigated and correlated in detail. On the contrary, not much effort has been spent on the mechanisms of indigo film growth, so the correlation between film morphology/crystallinity and charge transport is relatively unknown. In-depth studies of the structural and morphological characteristics of films of this material, also as a function of the deposition parameters, are therefore urgent to understand the growth processes and to improve the electrical performances of indigo-based devices.

As a pigment, indigo is highly insoluble in most solvents, a feature which makes the fabrication of the thin films by wet deposition methods difficult and dry physical deposition processes are usually preferred. In fact, the study of the films has displayed some complexities, ${ }^{27}$ and characteristics such 
as degree of crystallinity, polymorphism, morphology and molecular orientation at the solid interface, which all contribute to regulate the transport properties of a material, are far from being well understood. As for the transport properties, they were found to be dependent on the film growth substrate, ${ }^{27,28}$ with hydrocarbon-based materials such as polyethylene and tetratetracontane outperforming other dielectrics.

A first important issue to clarify concerns the solid phase present in the films. In its bulk crystal state, indigo is known to display two polymorphs (A and B) having very similar structures. ${ }^{29-34}$ Both forms are monoclinic with very similar unit cell parameters and we have recently shown that they grow as concomitant in most conditions. ${ }^{31}$ In a X-ray characterization of vapour deposited $40 \mathrm{~nm}$ thick indigo films on $\mathrm{Si} / \mathrm{SiO}_{2},{ }^{27}$ the specular scan showed only two reflections, which could be attributed equally well to weak crystallographic orientations along the $\left(\begin{array}{lll}1 & 0 & \overline{1}\end{array}\right)$ and $\left(\begin{array}{ll}2 & 1 \\ 2\end{array}\right)$ planes of crystal form A or (1 $\left.\begin{array}{lll}0 & 0\end{array}\right)$ and $\left(\begin{array}{lll}2 & 1 & 0\end{array}\right)$ of crystal form B. ${ }^{27}$ This left the film phase undetermined and prevented the detection of a possible concomitant polymorph growth. The actual orientation of the molecules relative to the substrate surface would be nearly the same regardless of the polymorph, but phase mixing would be anyway detrimental to charge transport. In another study, ${ }^{11}$ vapour grown films of different thicknesses and on various substrates were shown to display a sharp single diffraction peak, which was taken as an indication of a crystalline texture with a single preferential orientation, with no reference to a specific polymorph. Together with the usually observed $\left(\begin{array}{lll}1 & 0 & 0\end{array}\right)$ reflection, a new peak was detected in the GIWAX (grazing incidence wide angle x-ray scattering) pattern of a film grown on paraffin in Ref. 28 The feature was identified as due to a new crystal modification, described as a distorted monoclinic structure of polymorph A. Finally, films obtained by drop casting displayed only the presence of polymorph B when investigated by lattice phonon Raman spectroscopy. ${ }^{31}$ Concerning the morphology, indigo films evaporated on various dielectric materials from a hot-wall epitaxy source at a pressure of $1 \times 10^{-7}$ mbar were found uniform, continuous and highly crystalline on most substrates. ${ }^{11}$ Films $\approx 70 \mathrm{~nm}$ thick obtained by thermal evaporation at a pressure of $2 \times 10^{-6}$ mbar and a rate of $0.6 \AA /$ s displayed a very rough and non-continuous coating on Polyvinyl alcohol substrates and randomly distributed flakes on paraffin tetracontane, ${ }^{28}$ despite the fact that on the latter substrate the best OFET performances were observed. This suggested a dependence on the growth conditions and parameters, as well as on the nature of the interface. Lastly, when analysing the forces driving the formation and shaping the characteristics of the film crystal state, the role of strong intermolecular H-bonds has been either stressed ${ }^{11,27}$ or considered overestimated. ${ }^{28}$

Aiming to clarify the still open issues mentioned above, the present work deals with the study of structure and morphology of indigo films obtained by ultra-high vacuum deposition on $\mathrm{Si} / \mathrm{SiO}_{2}$ by using a combination of techniques and following a well established approach for indigoids 
semiconductors. ${ }^{35-37}$ After acting on the film morphology by modifying the conditions of growth and obtaining highly reproducible systems, we applied lattice phonon Raman microscopy and demonstrated that B is the only polymorph present in the film phase. Such an identification relies on the results of a study conducted on the indigo bulk crystals, which showed that the two known polymorphs can be easily recognized by this method. ${ }^{31}$

We gained further insights on the crystallization behaviour of indigo at the solid interface by using $\mathrm{X}$-ray diffraction (XRD), atomic force microscopy (AFM) and transmission electron microscopy and diffraction (TEM). The last technique showed that the molecular arrangement in the film is in fact driven by the formation of strong hydrogen bridging bonds, which determine the development of the structure along a preferential lattice direction, in which such interactions are exerted. Consequently, the crystallinity of the film was generally poor even though it could be improved with a solvent vapour annealing treatment.

\section{Materials and methods}

\subsection{Sample preparation}

Commercial indigo powder (TCI Chemicals, 97\%), entirely composed of polymorph B, was purified by double sublimation at $200{ }^{\circ} \mathrm{C}$ in low pressure of nitrogen to avoid thermal oxidation. The purification stage of the raw material is a crucial step for obtaining homogeneous and reproducible films, due to the high content of impurities of the commercial product. ${ }^{15} \mathrm{We}$ found that a double sublimation of the commercial powder in a cold finger apparatus produces highly pure microcrystals with both indigo polymorphs. As expected, the growth characteristics on the cold finger surface depend strongly on its temperature: ${ }^{38}$ the higher is the temperature of the cold finger, the higher is the quality of the obtained microcrystals. The largest and purest crystals were obtained for a cold finger temperature of $\approx 130-140{ }^{\circ} \mathrm{C}$, i.e. without the cooling fluid (water at RT). These crystals represented the optimal starting material for obtaining a stable flux of molecules, sublimed by means of a Knudsen cell in vacuum.

Indigo films were deposited in an Organic Molecular Beam Deposition (OMBD - base pressure of $2 \times 10^{-8}$ mbar) on $1 \mathrm{~cm}^{2}$ silicon substrates (2-10 $\Omega \cdot \mathrm{cm}, p$ doped, CZ 1-0-0) with a $100 \mathrm{~nm}$ thick layer of thermally grown silicon dioxide $\left(\mathrm{SiO}_{2}\right)$. A Quartz Micro-Balance (QMB), placed close to the substrate, was used to monitor the weight of molecules adsorbed on the substrate with 1ng of sensitivity. To avoid thermal drift effects on measurements, the QMB was kept at $20^{\circ} \mathrm{C}$ by means of a water cooler. Indigo films were grown by depositing a fixed amount of molecules, viz $1000 \mathrm{ng}$, by keeping the substrate at RT. The impinging flux, or deposition rate, $F$ of molecules coming from the 
Knudsen cell was varied from 10 to $200 \mathrm{ng} / \mathrm{min}$, specifically 10, 20, 42, 70, 101, 170 and 200 ng/min. Prior to insertion in the OMBD system, the $\mathrm{Si} / \mathrm{SiO}_{2}$ substrate was cleaned with acetone vapours to remove possible organic contaminants.

\subsection{X-ray diffraction}

For phase identification of thin film samples grown at various deposition speed (for the preparation see above) X-ray powder diffraction patterns (XRPD) were collected in the $2 \theta$ range between $5^{\circ}-40^{\circ}$, on a PANalytical X'Pert PRO automated diffractometer with Bragg-Brentano geometry equipped with an $\mathrm{X}^{\prime}$ Celerator detector, using $\mathrm{Cu} \mathrm{K} \alpha$ radiation without a monochromator (step size, $0.02^{\circ}$; time/step, $20 \mathrm{~s} ; 0.04$ rad soller; 40mA x 40kV). The program Mercury ${ }^{39}$ was used for the simulation of X-ray powder patterns on the basis of single crystal data retrieved from the Cambridge structural database $^{40}$ (with CSD refcodes MIXCEE and INDIGO02 for polymorphs A and B, respectively).

\subsection{Profilometer and AFM}

As reported above, indigo films were grown by depositing the same amount of molecules, i.e. 1000 $\mathrm{ng}$, corresponding to a constant films thickness of (36 \pm 9$) \mathrm{nm}$. Films thicknesses were measured by averaging the thickness of each film in three different step edge positions by means of a stylus profiler (P-7 KLA Tencor, Milpitas, California, USA).

Topographic images were collected by Atomic Force Microscopy (AFM, SOLVER HV-MFM, NTMDT Zelenograd, Moscow, RU) operating in intermittent contact by using HA_NC cantilevers (NTMDT, Cantilever A: $\omega_{0}=(235 \pm 10) \mathrm{kHz}$ and $k=(12 \pm 2) \mathrm{N} \cdot \mathrm{m}^{-1}, \omega_{0}=(140 \pm 10) \mathrm{kHz}$ and $k=(3.5 \pm$ $0.7) \mathrm{N} \cdot \mathrm{m}^{-1}$ ). Images were morphologically analysed for investigating the evolution of the films $v s F$. The quality of AFM images was initially hampered by unstable tip-sample interaction along the scanning path, possibly due to residual electrostatic charges or inhomogeneous surface adhesion. As reported elsewhere, ${ }^{41}$ stable and reproducible AFM images were obtained by immersing the films in deionized water for $30 \mathrm{~min}$.

\subsection{Raman spectroscopy}

Raman spectra in the energy interval of the lattice phonons were recorded with a Horiba Jobin Yvon T64000 spectrometer equipped with three monochromators in a double subtractive configuration. The spectrometer was coupled to an Olympus BX40 confocal microscope equipped with $100 \times, 50 \times, 20 \times$ and $10 \times$ objectives, for a lateral resolution lower than $1 \mu \mathrm{m}$ with the $100 \mathrm{x}$ objective. This allows gathering information on the polymorphic composition in crystal domains of micrometric 
dimensions. The excitation wavelength used was the $647.1 \mathrm{~nm}$ line from a tunable $\mathrm{Kr}^{+}$gas laser, with a nominal power of $1 \mathrm{~W}$. Raman spectra collected in the energy interval of the internal molecular vibrations (100-2000 $\mathrm{cm}^{-1}$ wavenumber range) were recorded on a single grating spectrometer Renishaw System 1000 equipped with a suitable notch filter and coupled to a Leica DM LM microscope. The microscope was equipped with 50x, 20x and 5x objectives. The excitation was from the $785 \mathrm{~nm}$ line of a diode laser with a nominal power of $500 \mathrm{~mW}$. In all cases, power was reduced by neutral density filters to avoid sample damage.

\subsection{Electron microscopy}

Transmission electron microscopy (TEM) measurements were performed, both in bright field (BF) and selected area electron diffraction (SAED) modes, by using a Philips CM12 TEM fitted with a LaB6 filament and equipped with a SIS Megaview III CCD camera. A flotation technique was adopted to transfer the films on the copper electron microscopy grids (200 mesh) for the subsequent TEM analysis, as described in the following. First, the indigo/ $/ \mathrm{SiO}_{2}$ samples were coated with a thin carbon film in an Edwards E306A coating system. Secondly, the carbon-coated films were removed from the $\mathrm{SiO}_{2}$ substrate by gently plunging the samples in a 5\% (w/w) $\mathrm{HF}$ acid solution in water. The films were retrieved with the copper grids directly from the HF solution, and then washed abundantly with MilliQ water. The samples subjected to solvent vapor annealing (SVA) of MeOH (Aldrich) were placed at room temperature on a sample holder in an airtight container with a reservoir of about 20 $\mathrm{ml}$ of solvent in a glass Petri dish for times variable between 8 and 24 hours.

For the electron diffraction (ED) measurements, the accelerating voltage was $120 \mathrm{kV}$ and, in order to minimize the beam damage, the low dose mode of the microscope was used, together with small $\mathrm{C} 2$ aperture $(5 \mu \mathrm{m})$ and low current (spot 5-7 setting and 24000x). The ED patterns were recorded at a $2.05 \mathrm{~m}$ chamber length using a $5 \mu \mathrm{m}$ condenser (C2) aperture in order to have a near-parallel illumination, about $50 \mu \mathrm{m}$ wide on the specimen. In Selected Area Electron Diffraction (SAED) mode an aperture of $100 \mu \mathrm{m}$ is inserted in the image plane in order to pick a specimen area of about 500 nm. For the analysis of the diffraction, we employed the Cerius 2 simulation package (Accelrys, Waltham, USA and Cambridge, UK). 


\section{Results and discussion}

\subsection{Polymorph characterization}

To check for the crystallinity and phase assignment of the films, a preliminary screening was performed by X-ray diffraction and Raman spectroscopy. In Figure 2a we show the patterns of samples grown at $F=10,70$ and $200 \mathrm{ng} / \mathrm{min}$, i.e. the lowest deposition rate, an intermediate rate, and the highest one. As already stated, this kind of measurement cannot determine unambiguously the crystalline form, which was thus identified as being polymorph B in all samples by Raman spectroscopy (vide infra).

The film deposited at the $200 \mathrm{ng} / \mathrm{min}$ rate does not show any detectable peak attributable to indigo, while those obtained at lower deposition rates, namely 70 and $10 \mathrm{ng} / \mathrm{min}$, display very similar diffraction patterns with two peaks: the first at $10.7^{\circ}$ (with FWHM of $0.42^{\circ}$ and $0.52^{\circ}$, respectively) corresponding to a d-spacing of $8.28 \AA$, and the second at $33^{\circ}$, due to the substrate. As described in Section 3.3 , the $10.7^{\circ}$ peak was revealed in the sample grown at $200 \mathrm{ng} / \mathrm{min}$ rate only after the SVA treatment. The peak position and the inter-plane distance agree with the previous determinations..$^{27,28}$ By combining this information with the finding by Raman spectroscopy (vide infra) and the crystal structure of form B, we can infer that the films are oriented alongside the $\left(\begin{array}{lll}1 & 0 & 0\end{array}\right)$ plane. The molecular orientation within this plane is shown in Figure $2 b$. The Scherrer equation ${ }^{42}$ was used to roughly estimate the average crystal size, which turn out to be 20 and $16 \mathrm{~nm}$ for the film obtained at $10 \mathrm{ng} / \mathrm{min}$ and $70 \mathrm{ng} / \mathrm{min}$, respectively.

a)

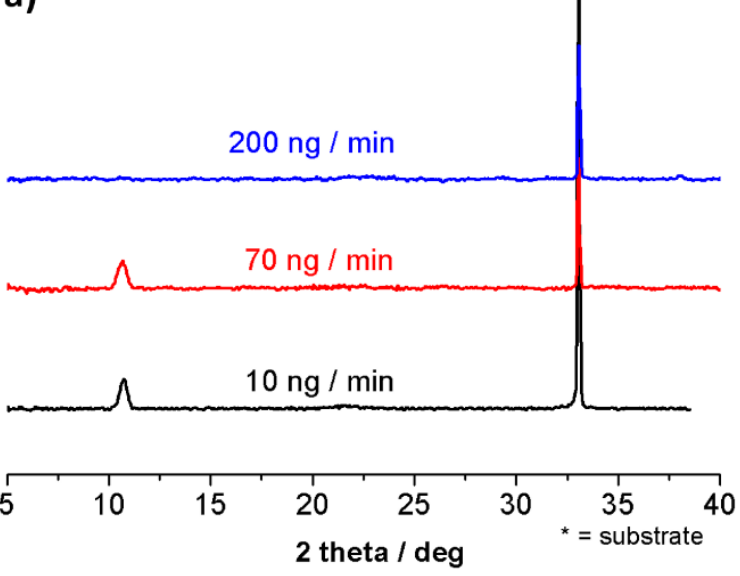

b)

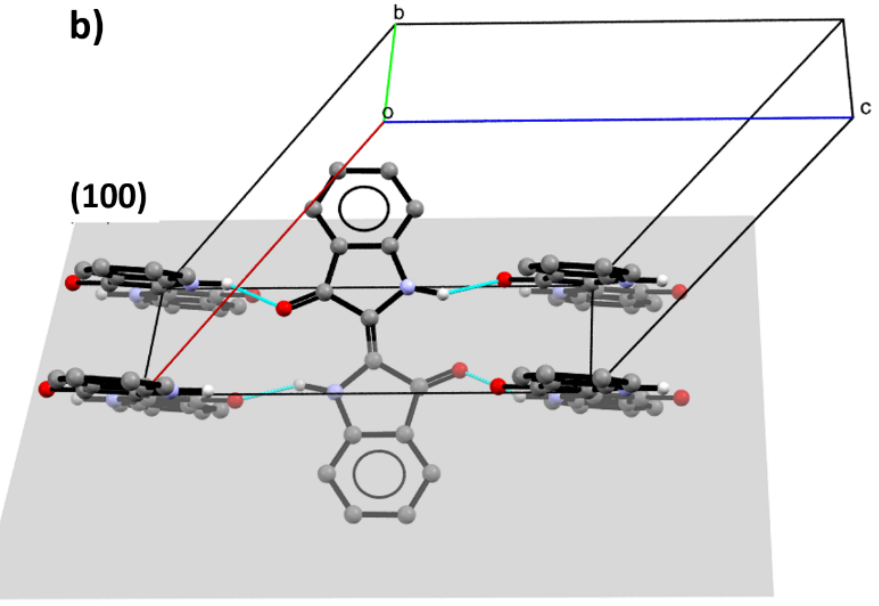

Figure 2: (a) XRD pattern of indigo thin films grown on Si/SiO2 wafer by sublimation at different deposition rates: 10 ng/min (black-line), $70 \mathrm{ng} / \mathrm{min}$ (red-line), and $200 \mathrm{ng} / \mathrm{min}$ (blue-line). (b) Orientation of the indigo molecules with respect to the (100) Miller plane. 
In Figure 3 we report the Raman spectra for the same films analysed by XRD. The spectral analysis relies on the previous characterization of indigo in bulk crystals. ${ }^{31}$ This showed how polymorphs A and B can be discriminated by their different spectra both in the lattice phonon region and, unexpectedly for packing polymorphs, by marker bands in the energy region of the molecular vibrations, due to their different patterns of intermolecular H-bonds (see Figure S1 and S2 in the SI). The lattice phonon region collected for all the films displays a broad band centred around $41 \mathrm{~cm}^{-1}$ and two more broad peaks at 92 and $102 \mathrm{~cm}^{-1}$. The spectral deconvolution of Figure 3 shows that the scattering is resolved into the contributions of the six lattice modes at 40, 42,74, 84,92 and $102 \mathrm{~cm}^{-1}$ $(\mathrm{FWHM}=7.5)$ that compose the pattern typical of polymorph B. This allow us to identify this structure as the one present in all samples, based on the results both of its single crystal spectrum and of powder samples reported in Ref. ${ }^{31}$ Note that the average bandwidth for the film is found to be 7.5 $\mathrm{cm}^{-1}$, against the value of $5.0 \mathrm{~cm}^{-1}$ of the single crystal, as the result of the inhomogeneous broadening expected in the polycrystalline sample.

The polymorph assignment is confirmed by the Davydov splittings at $132 / 139 \mathrm{~cm}^{-1}$ and at $1576 / 1587 \mathrm{~cm}^{-1}$ (Figure 3, left-hand side) which have been identified as the molecular modes lying at different wavenumbers in indigo polymorphs A and B. Spectra were collected at several spots of the films, detecting basically the same peaks, patterns and background, with no relevant changes in relative band intensities which would denote different crystalline orientations on the micrometre scale. The presence of the lattice phonon scattering in the sample grown at the $200 \mathrm{ng} / \mathrm{min}$ rate, revealing a degree of crystallinity which appears to go undetected in the X-ray measurements, is not surprising. The microscopic technique identifies crystalline domains and cannot average on largescale conditions such as the X-rays. 

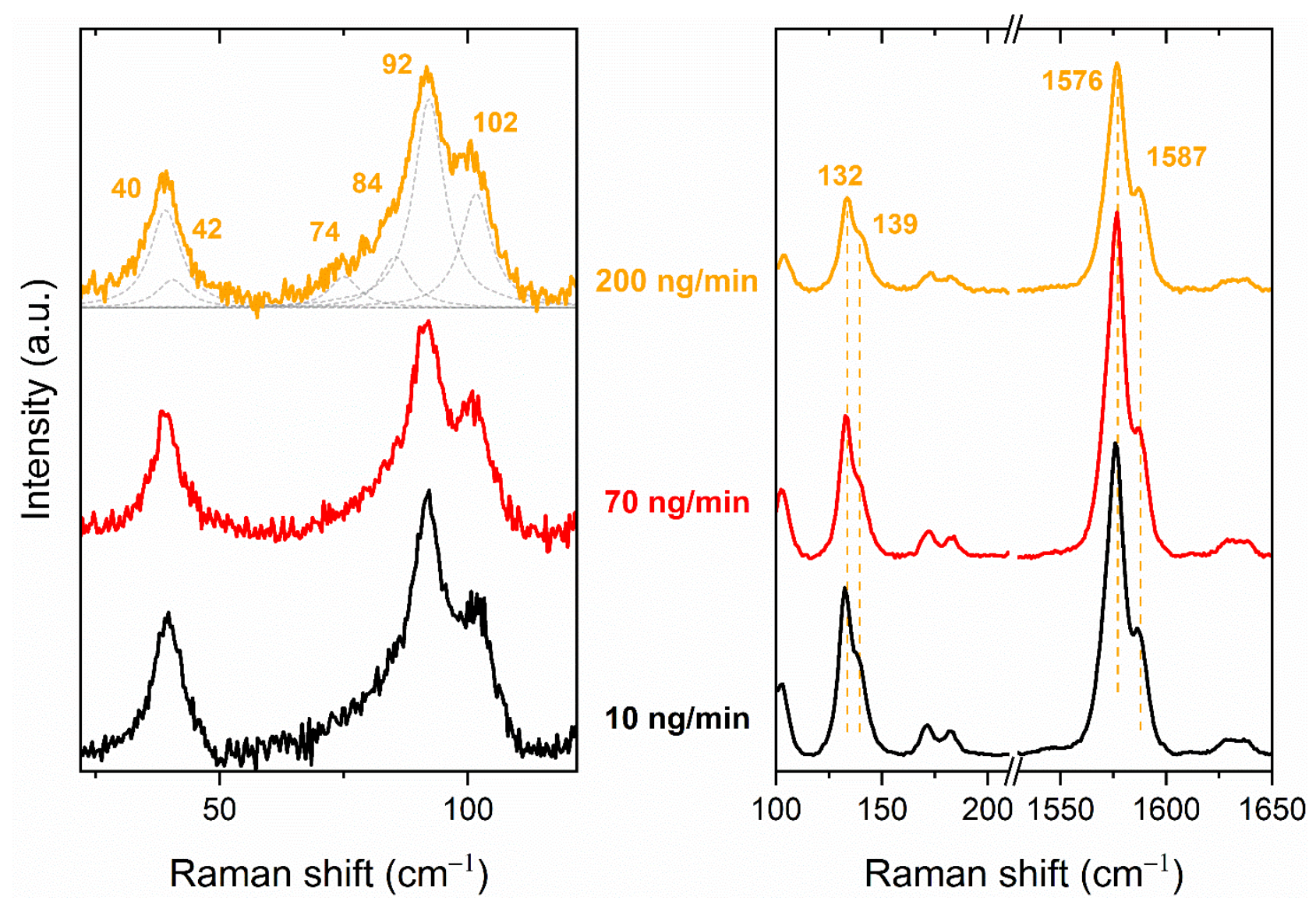

Figure 3: Micro Raman analysis of indigo film obtained at rate 10, 70 and $200 \mathrm{ng} / \mathrm{min}$. Left-side) Raman shifts (in wavenumbers) in the energy interval of the lattice phonons, and their deconvolution; Right-side) Raman shifts collected in the energy intervals of the internal molecular vibrations which allow for polymorph discrimination. Baseline was subtracted, intensities are in arbitrary units.

Thus, the picture given by Raman, is that of films appearing homogenous at the resolution allowed by the method and containing only one polymorph. Even though the analysis cannot provide information about molecular organization of the film, the moderate broadening of the lattice phonon peaks compared to those of the single crystal $^{31}$, as well as the general appearance, suggests that these are not the spectra of powders. ${ }^{31}$

\subsection{Elucidation of the crystal growth mechanism}

\section{Profilometer measurements and AFM analysis}

Contact profilometer measurements show a film thickness independent of the deposition rate, i.e. $1000 \mathrm{ng}$ of deposited molecules corresponds always to films $(36 \pm 9) \mathrm{nm}$.

As shown in AFM topographies of Figure 4, the films consist of 3D grains, that evolve progressively from large faced structures to spherical ones, for the lowest (10 $\mathrm{ng} / \mathrm{min})$ and the highest (200 $\mathrm{ng} / \mathrm{min})$ deposition rates $F$, respectively. For $F=10 \mathrm{ng} / \mathrm{min}$, the surface coverage $\theta$ of films are just above the percolation $\operatorname{limit}^{43}(\theta \approx 80 \%$, Figure $4 \mathrm{a})$, while the surface is fully covered for higher $F(\geq 20 \mathrm{ng} / \mathrm{min}$, Figure $4 \mathrm{~b}$ and $4 \mathrm{c}$ ). The surface roughness $\sigma$ of films, the average radius $R_{\text {avg }}$ of grains and their 
volumes $V_{\text {avg }}$ decrease exponentially for increasing $F$ (Figure $4 \mathrm{~d}$ and e, and SI, Figure S4), resulting into a morphological evolution of films associated to a surface smoothening of films which saturates for $F>100 \mathrm{ng} / \mathrm{min}$ (Figure $4 \mathrm{a}-\mathrm{c}$ ). Such results are similar to those obtained on perylene films and reported in the literature. ${ }^{44}$
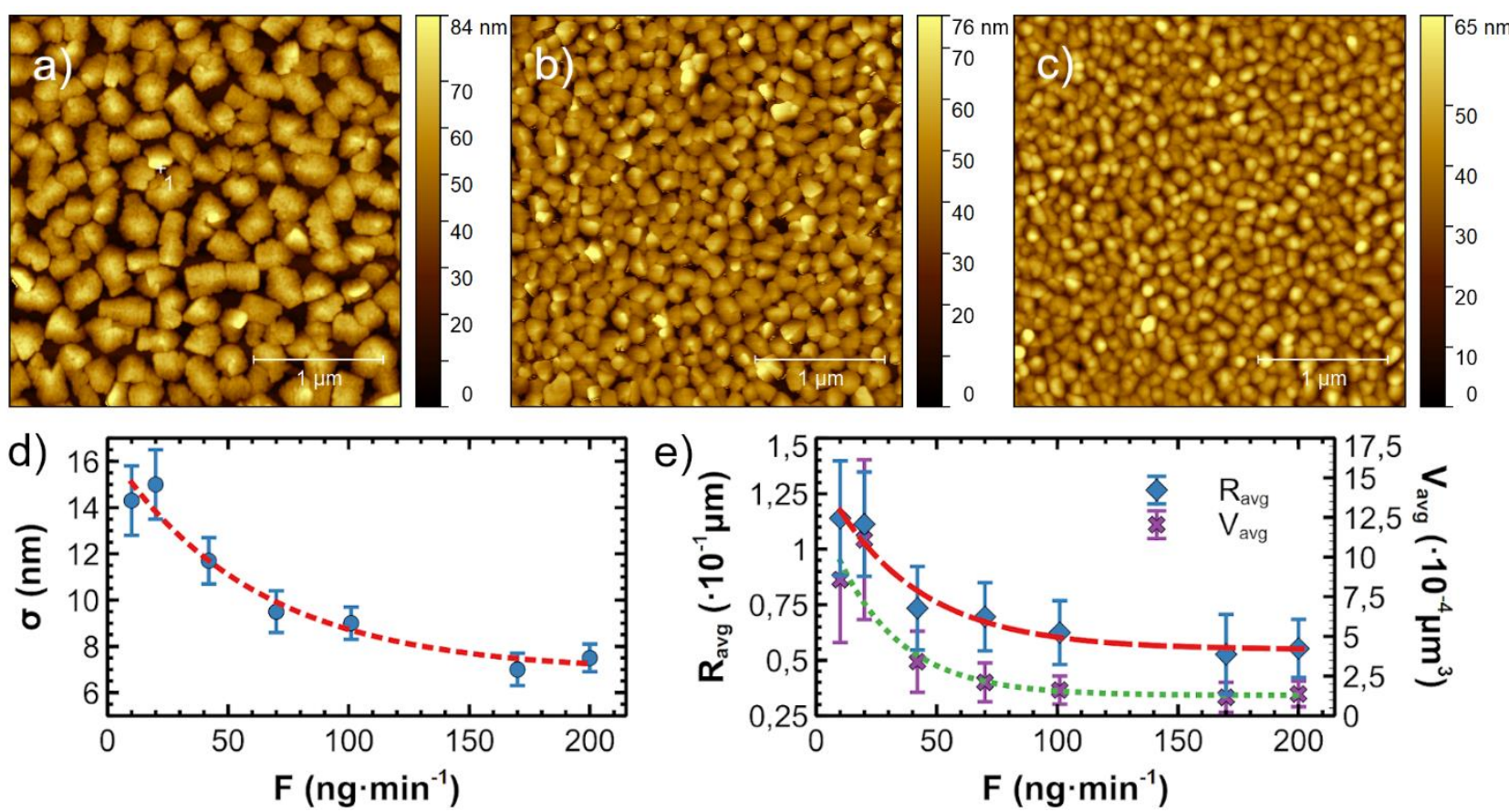

Figure 4: Topographic images of indigo films deposited on thermal $\mathrm{SiO}_{2}$ at room temperature for increasing deposition rates: $10(a), 70(b)$, and $200 \mathrm{ng} / \mathrm{min}(\mathrm{c})$. Images are $3 \times 3 \mathrm{\mu m}^{2}$. Surface roughness $\sigma(d)$ and the average grains radius $R_{\text {avg }}$ and the average volume $V_{\text {avg }}(e)$ decay exponentially (fitting lines) vs the deposition rate F. Independently of the deposition rate, the roughness analysis of images with different lateral sizes (e.g. from 40 to 1 um) highlights a roughness saturation above $5 \mu \mathrm{m} .{ }^{45}$ Accordingly, the evolution of all measured parameters $\left(\sigma, R_{\text {avg }}\right.$ and $\left.V_{\text {avg }}\right)$ were investigated by analysing $7 \times 7 \mu \mathrm{m}^{2}$ topographic images.

Indigo assembly on the $\mathrm{SiO}_{2}$ surface shows a Volmer-Weber growth mode (3D), unlike the Stranski-Krastanov growth mode $(2 \mathrm{D}+3 \mathrm{D})$ commonly obtained with small organic molecules deposited on $\mathrm{SiO}_{2}$ substrates by OMBD. ${ }^{46,47}$ The AFM images at the lower deposition rates (10 and $20 \mathrm{ng} / \mathrm{min}$ ) show islands developing in a 3D growth that do not expand along the $b c$ crystallographic plane parallel to the substrate. This growth is unforeseen because a low deposition rate should be favouring a single molecule adsorption process and a diffusion-mediated growth, thus yielding a layer-by-layer growth mode (2D) of films. ${ }^{48}$ In the literature, only picene grown on $\mathrm{SiO}_{2}$ shows a similar growth mode for which the Volmer-Weber growth mode prevails regardless of the film thickness. $^{49-51}$

Such morphological evolution of indigo films seems to follow the theory of grain coalescence suppression introduced by Lü $e t a l^{52}$ for interpreting the growth of Ag grains vapor deposited on 
$\mathrm{SiO}_{2}{ }^{53}$ Within the framework of this theory, the growth of Ag grains resembles a coalescence-free growth at room temperature. This seems in agreement to AFM images where the grains density $N$ (i.e. number of grains per unit surface $-\# / \mu \mathrm{m}^{2}$ ) increases largely for increasing $F$ (see Figure $4 \mathrm{a}-$ c) ${ }^{54}$ In the case of homogeneous nucleation, the density of stable islands $N$ is expected to vary as a power law $F^{\delta}$ with the deposition rate, with $\delta$ being the power law exponent. ${ }^{55}$ Different regimes of condensation (complete, initially incomplete and totally incomplete) lead to different power laws for $N$ as a function of $F$. In our experimental conditions (low temperature, being the substrate kept at $\mathrm{RT})$, the regime is a complete condensation of 3D grains. Accordingly, $\delta=i \cdot(i+2.5)^{-1}$ where $i$ is the critical nucleus size, i.e. the minimum number of molecules forming a stable nucleus on the surface. ${ }^{55}$ The power law exponent $\delta$ for indigo is $(0.56 \pm 0.05)$ and corresponds to a critical nucleus size $i$ of 3 , in agreement with the results obtained for pentacene, ${ }^{48} \mathrm{Alq}_{3}{ }^{56,57}$ and hexathiophene. ${ }^{58}$ By considering that $N$ is determined by the coalescence of islands at the begin of the growth and it is independent of the film thickness, ${ }^{59}$ the coalescence-free growth scenario seems reasonable.
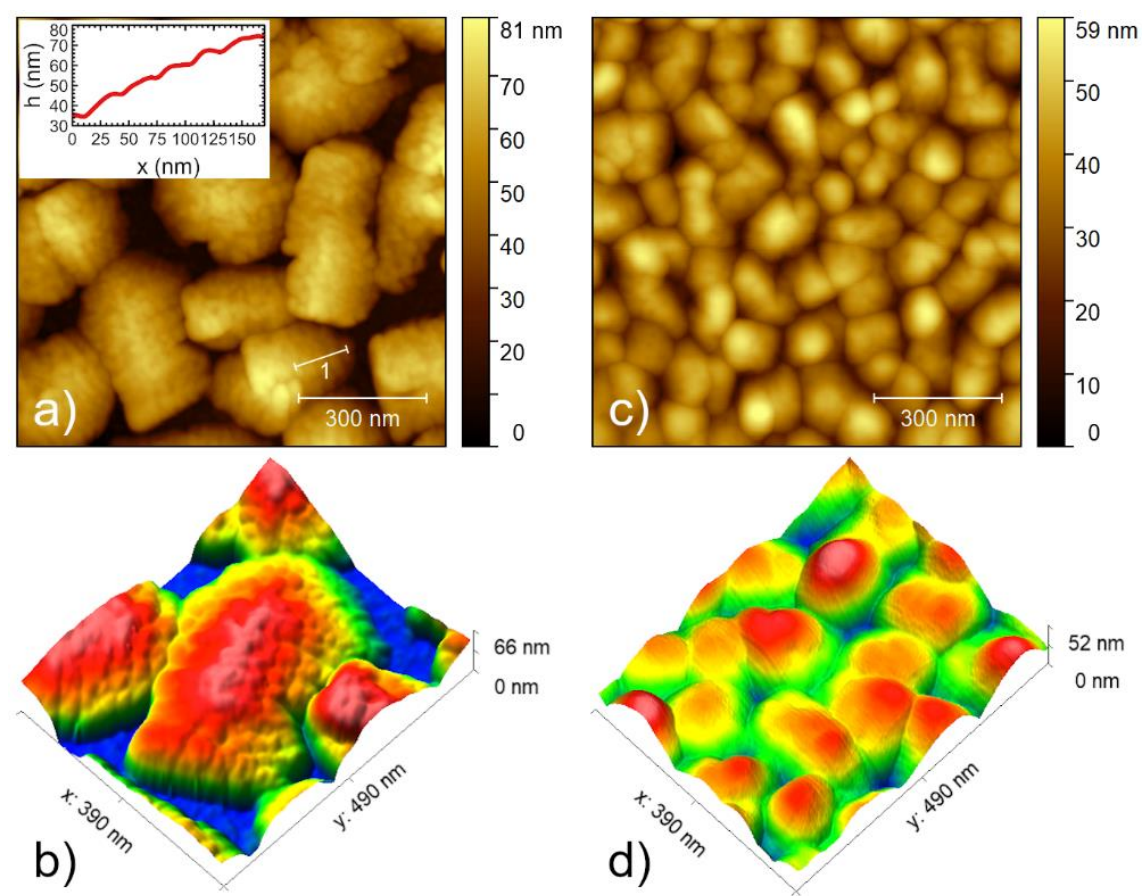

Figure 5: Topographic images of typical indigo films grown at $10\left(\text { a) and } 200 \text { (c) } \mathrm{ng} / \mathrm{min} \text { (size } 1 \mu^{2}\right)^{2}$. 3D view of a large faced structure and few grains of films grown at $10($ b) and $200(d) \mathrm{ng} / \mathrm{min}$ (cropped images). Inset: Illustrative stepped height taken along the line 1 indicated in Figure (a).

Three-dimensional grains were investigated at higher magnification for the two limit cases: 10 and $200 \mathrm{ng} / \mathrm{min}$. The films deposited at the lowest deposition rate are composed of large faced structures exposing the $\mathrm{SiO}_{2}$ surface (darkest regions in Figure 5a). The structures show a stepped height profile with an average step of $\approx 10 \mathrm{~nm}$ (see inset of Figure 5a) and they are composed of grains with an 
average diameter $D=(30 \pm 10) \mathrm{nm}$, as calculated statistically from the radial autocorrelation function performed on $1 \times 1 \mu \mathrm{m}^{2}$ topographic images ${ }^{60}$ (see Figure $5 \mathrm{~b}$ ). The average diameter of grains on such large faced structures is equal, within the experimental error, to the average diameter obtained by TEM analysis (see Figure S3 and S5 in the SI). It is not possible with topographic AFM images to discern if grains, possibly formed by instabilities of molecular films (like post-deposition wetting ${ }^{61}$ ) are only on the surface, or also within the structure. However, the average step width (see inset of Figure 5a) is similar to the average diameter of grains, suggesting possible re-organization phenomena effects. Films deposited at the highest deposition rate are composed of spherical grains with an average diameter $D=(120 \pm 20) \mathrm{nm}$ (Figure $5 \mathrm{c}$ and d).

\section{TEM measurements}

TEM analysis was carried out on the three most representative samples $(10,70$ and $200 \mathrm{ng} / \mathrm{min})$ both in bright field (BF) and electron diffraction (ED) modes. Figure 6 compares the nanomorphology of the samples analysed by AFM and TEM.
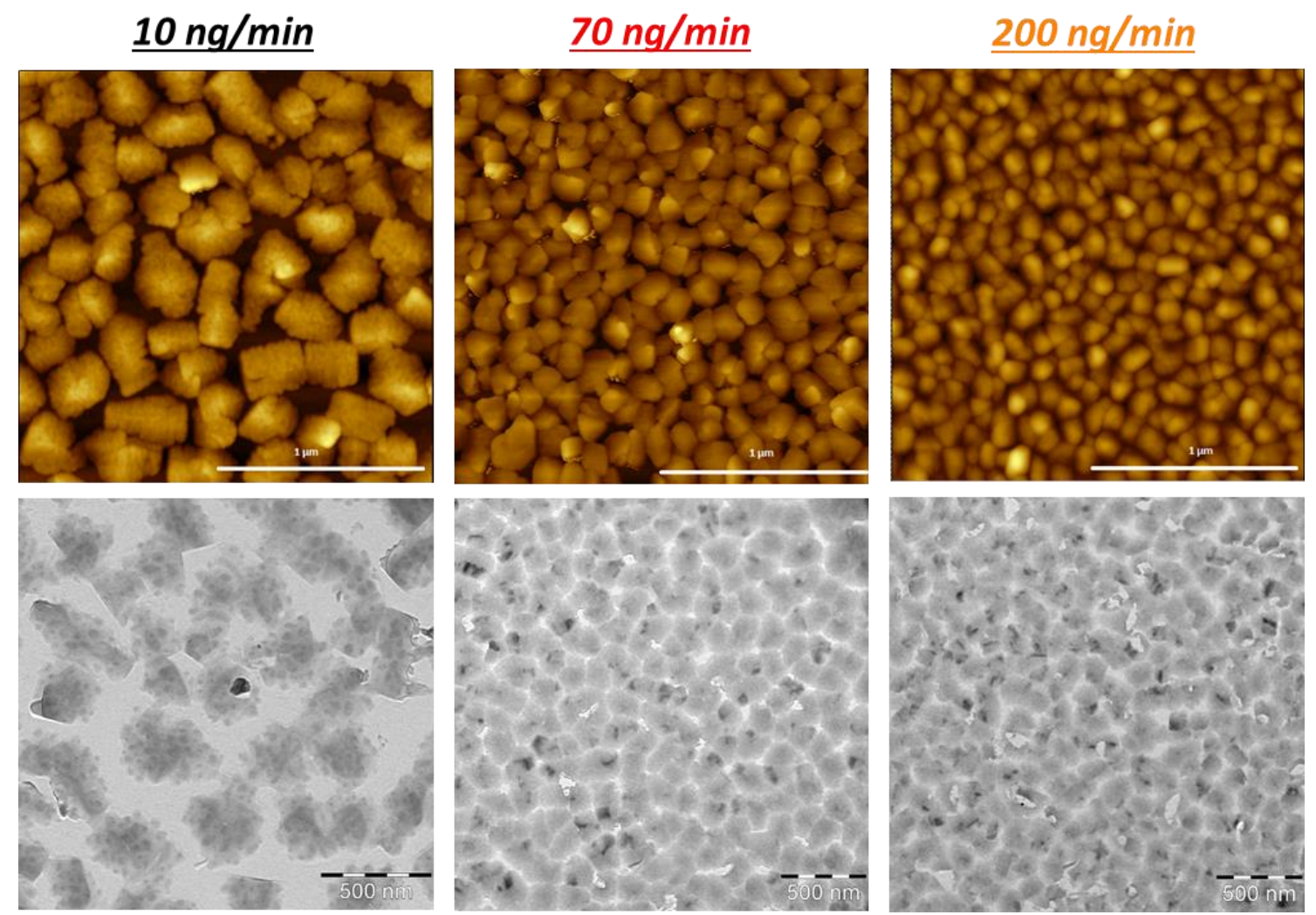

Figure 6: Comparison between AFM analysis (on the top) and TEM measurements in bright field (on the bottom) performed on the indigo film on silicon wafer.

The BF image of the $10 \mathrm{ng} / \mathrm{min}$ sample clearly reveals that the grains detected by AFM are not single crystals, even when they appear to have neat edges. They are instead made of smaller crystalline 
domains of irregular shape, embedded in each grain and identified by a sharper contrast in Figure 6. The much smaller grains of the films deposited at $70 \mathrm{ng} / \mathrm{min}$ and $200 \mathrm{ng} / \mathrm{min}$ are defined by the brighter boundaries in the BF image of Figure 6, and also here at higher resolution the presence of nanosized subdomains is obvious. The results of AFM and TEM on the average dimensions of the grains are also in agreement, as shown by the TEM data reported in SI (see Figure S5).

The evolution of the ED pattern as a function of the deposition rate is displayed in Figure 7 for the three representative samples, where both the SAEDs, large area ED and the corresponding averaged radial profiles of large-scale ED are reported. At the lowest deposition rate, we observe a set of few, sharp reflections in the SAED, which increase on increasing rate, finally evolving into the almost continuous ring of a highly polycrystalline sample. In particular, in the SAED (Figure 7 at $10 \mathrm{ng} / \mathrm{min}$ ) only one fragment of indigo is selected. The crystals exhibit a twinning structure and the SAED pattern can be thoroughly indexed as three twin domains oriented along the [2 111$]$ axis, as specified in Figure S6 in SI. 


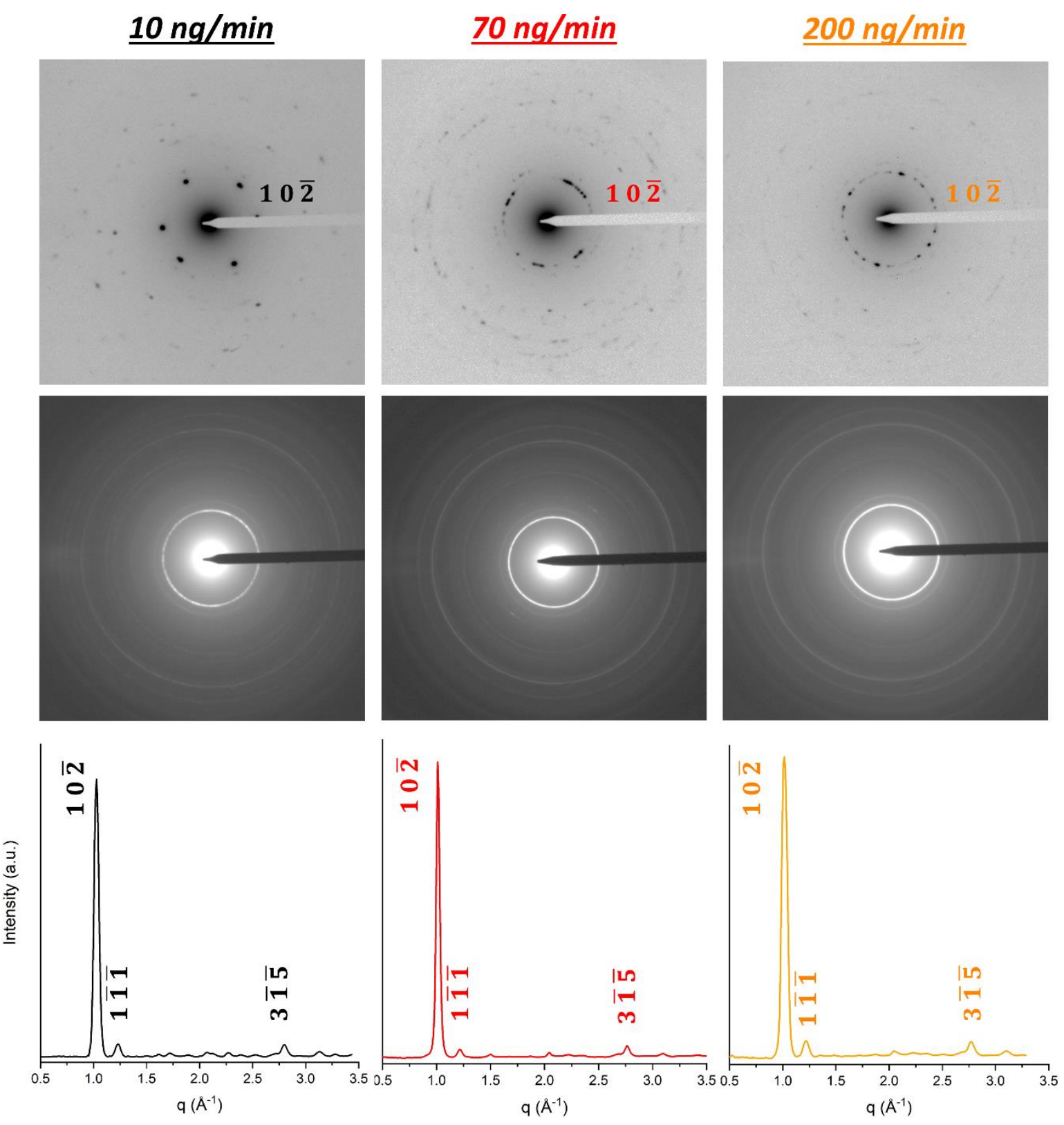

Figure 7: The Figure shows a comparison between the three samples in SAED (on the top), large area (about $50 \mu m$ ) ED in the centre and the corresponding radial averaged profile (intensity in arbitrary units) evidencing the major reflections at the bottom.

This result agrees with the observation of larger crystal domains in the low rate grown sample. One reflection is clearly dominant in all cases and corresponds to an inter-planar distance of $0.611 \mathrm{~nm}$, that can be indexed as $10 \overline{2}$ based on the B polymorph structure. The large area ED patterns highlight the presence and the evolution of the dominant Scherrer rings indexes $10 \overline{2}$ and $1 \overline{1} \overline{1}$ in all the films versus deposition rate. Overall, the ED patterns show that even when larger crystallites are formed, the film still display limited order given the few reflections seen in the ED patterns. However, ED indicates that the domains have a preferred growth direction, as suggested by the prevailing $10 \overline{2}$ reflection. The picture emerging is that in the samples deposited at lowest rate, the first layers produce 
a quasi-uniform film on which fragments of poorly aligned and much smaller crystallites with random orientation grow. This results in the polycrystalline pattern observed in the large area ED, while the SAED measurements, selecting single fragments, identify well defined orientations.

\subsection{Effect of recrystallization by solvent vapor annealing}

The vacuum deposited films show rather limited crystal dimensions and grains are formed by nanosized subdomains, resulting in overall poor crystallinity. For this reason, we used solvent vapor annealing as a potential method to increase the size of crystalline domains and improve overall crystallinity of thin films. In particular, the use of H-bonding solvents is appealing since it could potentially help re-crystallize the indigo subdomains.

Therefore, the grids were subjected to solvent vapor annealing with methanol for $24 \mathrm{~h}$, as this method has proven to be very effective in controlling crystallization while avoiding the destruction of the material. ${ }^{62,63}$ Among various tested solvents, methanol proved particularly effective. Upon SVA, a thin layer of solvent is adsorbed at the film surface and gives mobility to the indigo surface molecules that can re-organize in a process that is called Ostwald ripening whereby surface admolecules of indigo can diffuse from the smaller to the larger crystals. In the present case, the ripening mechanism allows for some reorganization of the small nanodomains into a larger domain with better defined crystallographic orientation.

Following the treatment, all the films displayed a modification of the ED patterns which indicated molecular re-organization. For instance, a SAED (Figure S7) recorded on the $10 \mathrm{ng} / \mathrm{min}$ film after SVA could be indexed and simulated by assuming a [4 1 12 ] zone axis of polymorph B, thus definitely confirming the phase assignment.
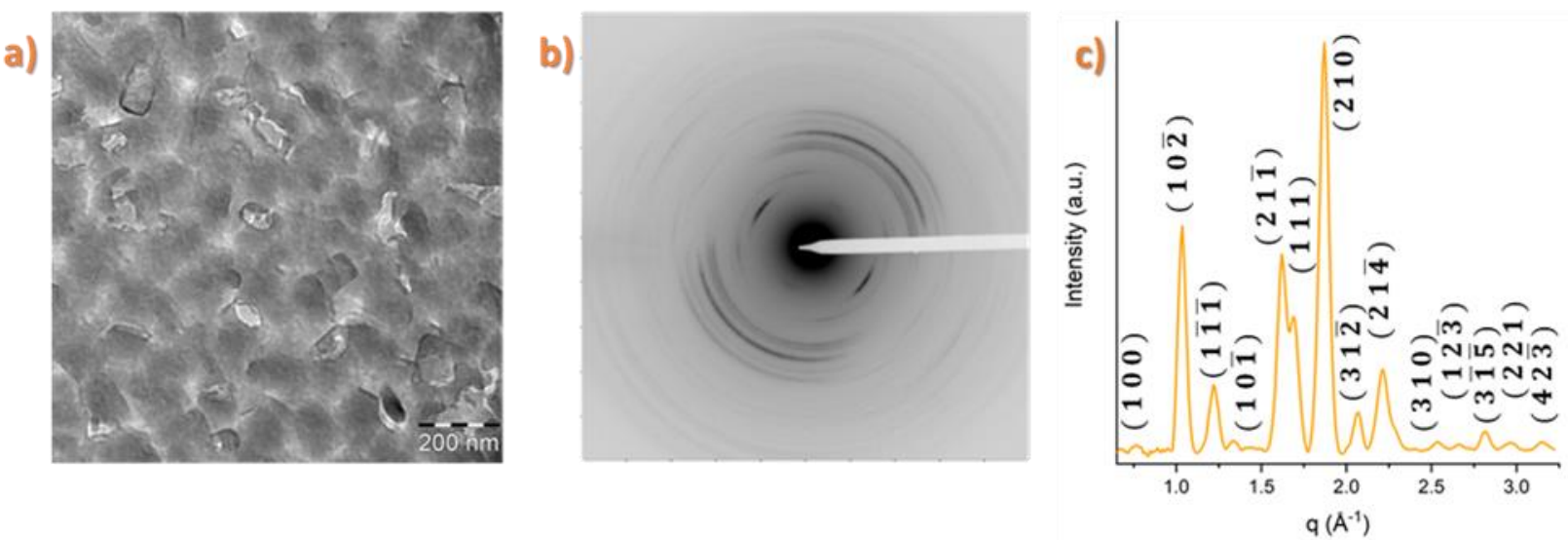

Figure 8: a) BF image performed on $200 \mathrm{ng} / \mathrm{min}$ Indigo film after SVA treatment; $b$ ) ED pattern of the SVA treated sample, c) Averaged section profiles of large-scale made on the ED shown in $b$ ). 
To describe in detail the effect of SVA, we focus on the $200 \mathrm{ng} / \mathrm{min}$ sample for which changes were most pronounced (Figure 8). After the SVA treatment, the nano-subdomains seen in the indigo grains of the pristine films are no longer visible. Indigo grains show better defined edges and the surface of the crystals display terraces. Accordingly, SVA promotes Ostwald ripening whereby small Indigo subdomains progressively merge and grow into larger domains with a better-defined crystalline orientation. Improved crystallinity is manifested by the appearance of numerous additional reflections. In Figure 8c, where an angular averaged ED pattern of the SVA treated film is shown, it appears that the number of reflections has increased, indicating re-crystallization upon the SVA process. . It is worth noting that the untreated film did not display any peak in the X-ray analysis of

a)

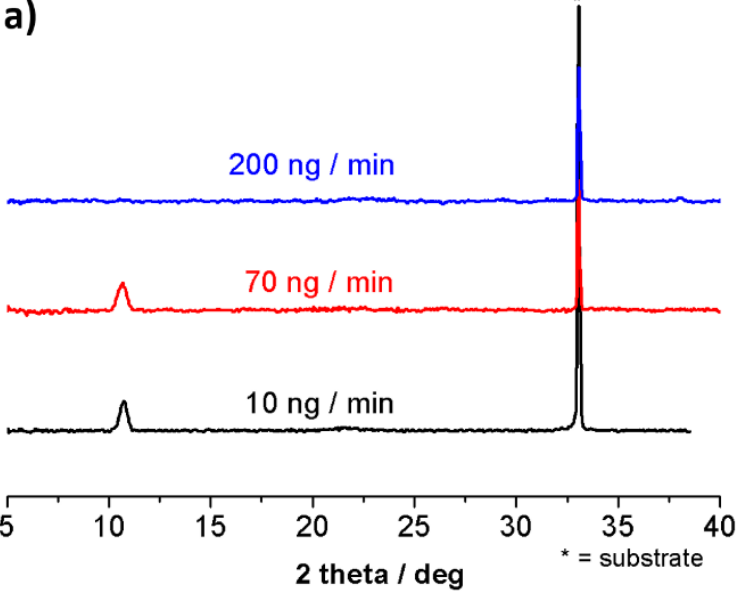

b)

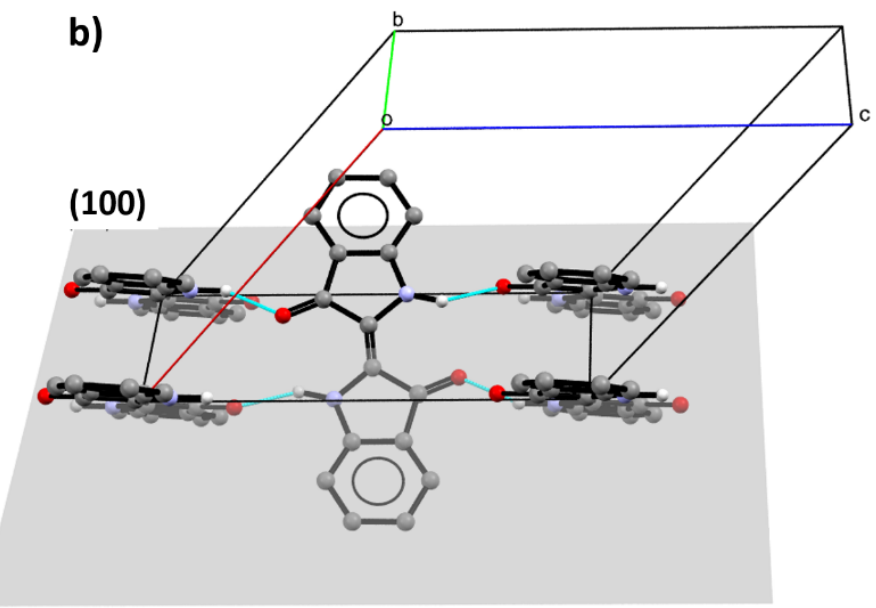

Figure 2, whereas now the (1 00 0) reflection is clearly detectable (Figure S8). However, the SAED patterns have not substantially improved in terms of intensity and number of well-defined reflections. Just as with the pristine films, the previously observed $10 \overline{2}$ is still predominant.
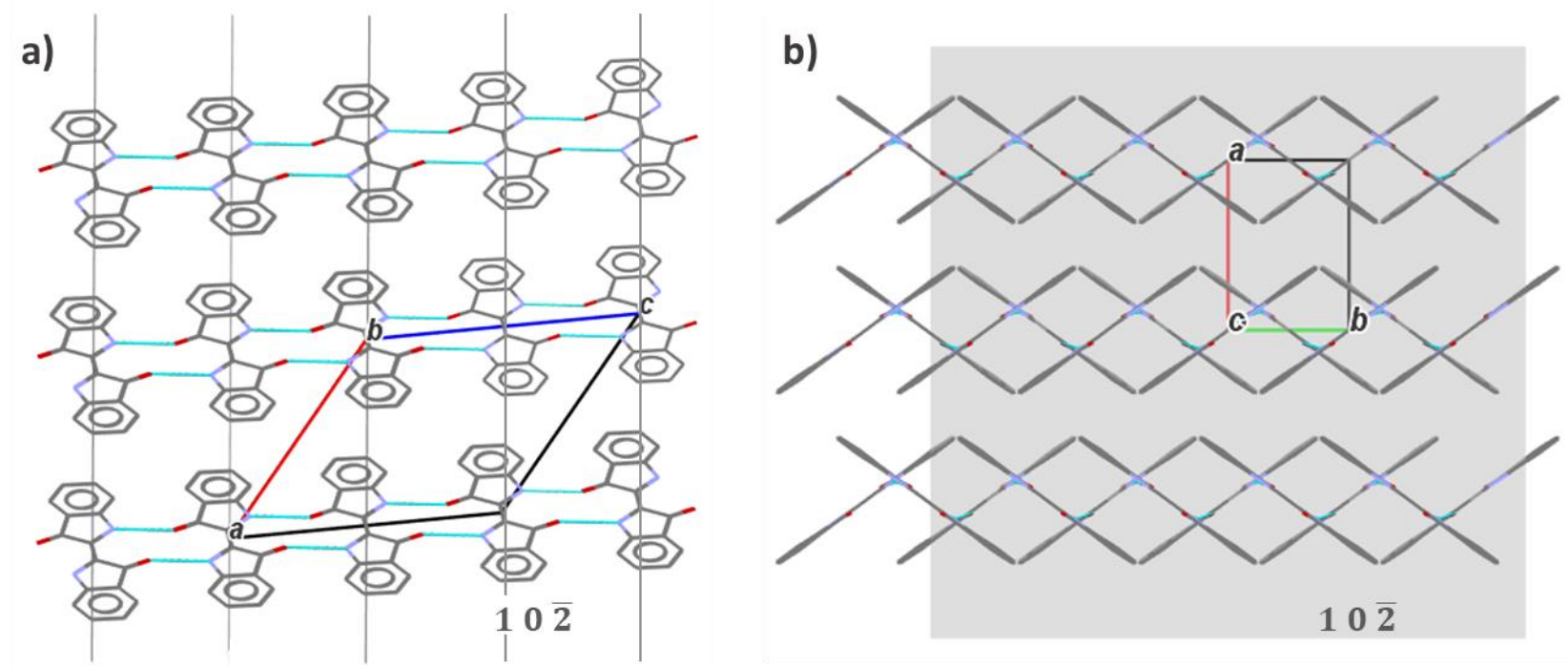

Figure 9: Two characteristic projections of the unit cell of indigo along the b axis (a) and along the H-bonding direction (c axis) (b) H-bonds are highlighted in light blue. The $\left(\begin{array}{lll}1 & 0 & \overline{2}\end{array}\right)$ plane is shown in light grey in $(b)$. 
Why is this reflection so predominant in indigo? As can be seen in Figure 9, the indigo molecules in two successive $\left(\begin{array}{lll}1 & 0 & \overline{2}\end{array}\right)$ planes are separated by a $0.611 \mathrm{~nm}$ distance, with the H-bond interactions characterizing the indigo solid state acting essentially along the $c$ axis, i.e. the direction perpendicular to the $\left(\begin{array}{lll}1 & 0 & \overline{2}\end{array}\right)$ plane. The direction of fast growth along $c$ is thus determined by such interactions, whereas growths in other lattice directions appear to be hampered. This explains why the reflections from other crystallographic planes are weak or absent in the ED pattern of the pristine films. The SVA treatment is a a way of enhancing the film crystallinity at least to a certain extent, as clarified by the ED pattern of Figure 8. The treatment results in the formation of a fibre-like film structure with the preferential direction of growth still being the $c$ axis. However, the section profile of the film reveals numerous new reflections, supporting a recrystallization upon SVA, with the growth of the crystals also along new lattice directions. Methanol is possibly effective as it is a H-bonding solvent that can break/screen the H-bond interactions, thus promoting the growth of the crystals in directions in which the bonding is absent. Despite its effectiveness, SVA cannot however produce large singlecrystal like domains. This is inherent to the fact that the strong unidirectional H-bonding in the indigo crystal induces a frustration of 3D crystallization.

\section{Conclusions}

As already reported in the literature, ${ }^{2,11-15,27,28}$ material purity, molecular packing, degree of crystallinity, growth method and parameters are crucial in the formation of indigo films. However, the influence of the deposition conditions on the film morphology, the probe of its crystallinity and the molecular organization at the substrate interface have not yet received the attention they deserve. This work, thanks to the availability of highly homogeneous and reproducible films from vacuum deposition of highly chemically pure material, represents a step forward in understanding these effects.

AFM topographic images show a morphological evolution of 3D grains over the range of the tested deposition rates. Raman measurements prove that the films are composed only of the polymorph B, ending the ambiguities reported in the literature on the indigo form which grows on silicon oxide substrates. This result indicates a full selectivity of the surface towards one of the two known polymorphs. In agreement with the literature, ${ }^{27,28} \mathrm{X}$-ray measurements suggest an average molecular arrangement of crystallites in the $\left(\begin{array}{lll}1 & 0 & 0\end{array}\right)$ plane. Both TEM and AFM measurements evidence a granular film structure; in particular, the large crystallites obtained at $F=10 \mathrm{ng} / \mathrm{min}$ are composed of small grains with an average diameter $D \approx 35 \mathrm{~nm}$, that are replaced by larger grains $(D \approx 140 \mathrm{~nm})$ for 
the highest $F$, i.e. $200 \mathrm{ng} / \mathrm{min}$. Regardless of $F$, grains develop along the $\left(\begin{array}{lll}1 & 0 & \overline{2}\end{array}\right)$ plane, as probed by TEM experiments, showing that intermolecular H-bonds are driving the growth of the indigo domains.

The full characterization of indigo films reported here for the first time opens the perspective of controlling the growth of films deposited on different substrates and possibly to modulate the molecular orientation at the interface. ${ }^{28}$ Moreover, such engineering of substrate/molecules interaction could be useful for a deeper understanding of the charge trasport mechanisms through the film, from which a proper chemical funcionalization could be derived. It has been noted, for instance, that intramolecular H-bonds are crucial for the exhibition of field-effect behavior in indigoids, while intermolecular hydrogen-bonds in fact contribute only to their insolubility. ${ }^{64}$

\section{Acknowledgments}

Authors are very grateful to Dr. Andrea Migliori for helpful discussions and valuable support in data analysis and interpretation. Furthermore, the authors would like to thank Federico Prescimone for giving access to the profilometer, and Paolo Mei and Tiziano Bonfiglioli for their technical support. SPM images were processed with the the software Gwyddion, ${ }^{65}$ morphological data were analysed by the GNU General Public Licensed software QtiPlot and some Figures are prepared by the GNU General Public Licensed software Veusz. AFM images were collected in the SPM@ISMN facility. The authors also thank the Microscopy platform of Institut Charles Sadron for the technical support in TEM. This work was supported by the MIUR-PRIN project "From natural to artificial light-harvesting systems: unveiling fundamental processes towards a bio-inspired materials design" (HARVEST) protocol 201795SBA3.

\section{References}

(1) Allwood, J. M.; Ashby, M. F.; Gutowski, T. G.; Worrell, E. Material Efficiency: A White Paper. Resour. Conserv. Recycl. 2011, 55 (3), 362-381. https://doi.org/10.1016/j.resconrec.2010.11.002.

(2) Głowacki, E. D.; Voss, G.; Sariciftci, N. S. 25th Anniversary Article: Progress in Chemistry and Applications of Functional Indigos for Organic Electronics. Adv. Mater. 2013, 25 (47), 6783-6800. https://doi.org/10.1002/adma.201302652.

(3) Guo, X.; Baumgarten, M.; Müllen, K. Designing $\pi$-Conjugated Polymers for Organic Electronics. Prog. Polym. Sci. 2013, 38 (12), 1832-1908. https://doi.org/10.1016/j.progpolymsci.2013.09.005.

(4) Hedley, G. J.; Ruseckas, A.; Samuel, I. D. W. Light Harvesting for Organic Photovoltaics. Chem. Rev. 2017, 117 (2), 796-837. https://doi.org/10.1021/acs.chemrev.6b00215. 
(5) Leo, K. Organic Photovoltaics. Nat. Rev. Mater. 2016, 1 (8). https://doi.org/10.1038/natrevmats.2016.56.

(6) Sirringhaus, H. 25th Anniversary Article: Organic Field-Effect Transistors: The Path beyond Amorphous Silicon. Adv. Mater. 2014, 26 (9), 1319-1335. https://doi.org/10.1002/adma.201304346.

(7) Quinn, J. T. E.; Zhu, J.; Li, X.; Wang, J.; Li, Y. Recent Progress in the Development of N-Type Organic Semiconductors for Organic Field Effect Transistors. J. Mater. Chem. C 2017, 5 (34), 8654-8681. https://doi.org/10.1039/C7TC01680H.

(8) Irimia-Vladu, M.; Troshin, P. A.; Reisinger, M.; Shmygleva, L.; Kanbur, Y.; Schwabegger, G.; Bodea, M.; Schwödiauer, R.; Mumyatov, A.; Fergus, J. W.; Razumov, V. F.; Sitter, H.; Sariciftci, N. S.; Bauer, S. Biocompatible and Biodegradable Materials for Organic Field-Effect Transistors. Adv. Funct. Mater. 2010, 20 (23), 4069-4076. https://doi.org/10.1002/adfm.201001031.

(9) Irimia-Vladu, M.; Glowacki, E. D.; Sariciftci, N. S.; Bauer, S. Green Materials for Electronics; Wiley-VCH, 2017.

(10) Irimia-Vladu, M. "Green" Electronics: Biodegradable and Biocompatible Materials and Devices for Sustainable Future. Chem. Soc. Rev. 2014, 43 (2), 588-610. https://doi.org/10.1039/C3CS60235D.

(11) Irimia-Vladu, M.; Głowacki, E. D.; Troshin, P. A.; Schwabegger, G.; Leonat, L.; Susarova, D. K.; Krystal, O.; Ullah, M.; Kanbur, Y.; Bodea, M. A.; Razumov, V. F.; Sitter, H.; Bauer, S.; Sariciftci, N. S. Indigo - A Natural Pigment for High Performance Ambipolar Organic Field Effect Transistors and Circuits. Adv. Mater. 2012, 24 (3), 375-380. https://doi.org/10.1002/adma.201102619.

(12) Zhang, M.; Bao, W. X.; Liu, X. L.; Yu, B. Z.; Ren, Z. Y.; Bai, J. T.; Fan, H. M. Large-Scale Synthesis of Porous Graphene through Nanoscale Carbothermal Reduction Etching. J. Mater. Sci. 2015, 50 (24), 78757883. https://doi.org/10.1007/s10853-015-9309-1.

(13) Głowacki, E. D.; Irimia-Vladu, M.; Kaltenbrunner, M.; Gsiorowski, J.; White, M. S.; Monkowius, U.; Romanazzi, G.; Suranna, G. P.; Mastrorilli, P.; Sekitani, T.; Bauer, S.; Someya, T.; Torsi, L.; Sariciftci, N. S. Hydrogen-Bonded Semiconducting Pigments for Air-Stable Field-Effect Transistors. Adv. Mater. 2013, 25 (11), 1563-1569. https://doi.org/10.1002/adma.201204039.

(14) Głowacki, E. D.; Irimia-Vladu, M.; Bauer, S.; Sariciftci, N. S. Hydrogen-Bonds in Molecular Solids - from Biological Systems to Organic Electronics. J. Mater. Chem. B 2013, 1 (31), 3742. https://doi.org/10.1039/c3tb20193g.

(15) Irimia-Vladu, M.; Kanbur, Y.; Camaioni, F.; Coppola, M. E.; Yumusak, C.; Irimia, C. V.; Vlad, A.; Operamolla, A.; Farinola, G. M.; Suranna, G. P.; González-Benitez, N.; Molina, M. C.; Bautista, L. F.; Langhals, H.; Stadlober, B.; Głowacki, E. D.; Sariciftci, N. S. Stability of Selected Hydrogen Bonded Semiconductors in Organic Electronic Devices. Chem. Mater. 2019, 31 (17), 6315-6346. https://doi.org/10.1021/acs.chemmater.9b01405. 
(16) Klauk, H. Organic Thin-Film Transistors. Chem. Soc. Rev. 2010, 39 (7), 2643. https://doi.org/10.1039/b909902f.

(17) Yuan, Y.; Giri, G.; Ayzner, A. L.; Zoombelt, A. P.; Mannsfeld, S. C. B.; Chen, J.; Nordlund, D.; Toney, M. F.; Huang, J.; Bao, Z. Ultra-High Mobility Transparent Organic Thin Film Transistors Grown by an offCentre Spin-Coating Method. Nat. Commun. 2014, 5 (1), 3005. https://doi.org/10.1038/ncomms4005.

(18) Tsutsui, Y.; Schweicher, G.; Chattopadhyay, B.; Sakurai, T.; Arlin, J.-B.; Ruzié, C.; Aliev, A.; Ciesielski, A.; Colella, S.; Kennedy, A. R.; Lemaur, V.; Olivier, Y.; Hadji, R.; Sanguinet, L.; Castet, F.; Osella, S.; Dudenko, D.; Beljonne, D.; Cornil, J.; Samorì, P.; Seki, S.; Geerts, Y. H. Unraveling Unprecedented Charge Carrier Mobility through Structure Property Relationship of Four Isomers of Didodecyl[1]Benzothieno[3,2- b ][1]Benzothiophene. Adv. Mater. 2016, 28 (33), 7106-7114. https://doi.org/10.1002/adma.201601285.

(19) Anthony, J. E.; Brooks, J. S.; Eaton, D. L.; Parkin, S. R. Functionalized Pentacene: Improved Electronic Properties from Control of Solid-State Order. J. Am. Chem. Soc. 2001, 123 (38), 9482-9483. https://doi.org/10.1021/ja0162459.

(20) Anthony, J. E. Functionalized Acenes and Heteroacenes for Organic Electronics. Chem. Rev. 2006, 106 (12), 5028-5048. https://doi.org/10.1021/cr050966z.

(21) Huang, C.; Barlow, S.; Marder, S. R. Perylene-3,4,9,10-Tetracarboxylic Acid Diimides: Synthesis, Physical Properties, and Use in Organic Electronics. J. Org. Chem. 2011, 76 (8), 2386-2407. https://doi.org/10.1021/jo2001963.

(22) Liscio, F.; Milita, S.; Albonetti, C.; D’Angelo, P.; Guagliardi, A.; Masciocchi, N.; Della Valle, R. G.; Venuti, E.; Brillante, A.; Biscarini, F. Structure and Morphology of PDI8-CN2 for n-Type Thin-Film Transistors. Adv. Funct. Mater. 2012, 22 (5), 943-953. https://doi.org/10.1002/adfm.201101640.

(23) Liscio, F.; Albonetti, C.; Broch, K.; Shehu, A.; Quiroga, S. D.; Ferlauto, L.; Frank, C.; Kowarik, S.; Nervo, R.; Gerlach, A.; Milita, S.; Schreiber, F.; Biscarini, F. Molecular Reorganization in Organic Field-Effect Transistors and Its Effect on Two-Dimensional Charge Transport Pathways. ACS Nano 2013, 7 (2), 1257-1264. https://doi.org/10.1021/nn304733w.

(24) Takimiya, K.; Osaka, I.; Mori, T.; Nakano, M. Organic Semiconductors Based on [1]Benzothieno[3,2- b ][1]Benzothiophene Substructure. Acc. Chem. Res. 2014, 47 (5), 1493-1502. https://doi.org/10.1021/ar400282g.

(25) Kyndiah, A.; Cramer, T.; Albonetti, C.; Liscio, F.; Chiodini, S.; Murgia, M.; Biscarini, F. Charge Transfer and Percolation in C 60 /Pentacene Field-Effect Transistors. Adv. Electron. Mater. 2015, 1 (11), 1400036. https://doi.org/10.1002/aelm.201400036.

(26) Shehu, A.; Quiroga, S. D.; D’Angelo, P.; Albonetti, C.; Borgatti, F.; Murgia, M.; Scorzoni, A.; Stoliar, P.; Biscarini, F. Layered Distribution of Charge Carriers in Organic Thin Film Transistors. Phys. Rev. Lett. 
2010, 104 (24), 246602. https://doi.org/10.1103/PhysRevLett.104.246602.

(27) Scherwitzl, B.; Resel, R.; Winkler, A. Film Growth, Adsorption and Desorption Kinetics of Indigo on SiO 2. J. Chem. Phys. 2014, 140 (18), 184705. https://doi.org/10.1063/1.4875096.

(28) Anokhin, D. V.; Leshanskaya, L. I.; Piryazev, A. A.; Susarova, D. K.; Dremova, N. N.; Shcheglov, E. V.; Ivanov, D. A.; Razumov, V. F.; Troshin, P. A. Towards Understanding the Behavior of Indigo Thin Films in Organic Field-Effect Transistors: A Template Effect of the Aliphatic Hydrocarbon Dielectric on the Crystal Structure and Electrical Performance of the Semiconductor. Chem. Commun. 2014, 50 (57), 7639. https://doi.org/10.1039/c4cc02431a.

(29) Susse, P.; Wolf, A. A New Crystalline Phase of Indigo Enantiomer Resolution of Spiroketals. Naturwissenschaften 1980, 67, 3400.

(30) Kettner, F.; Hüter, L.; Schäfer, J.; Röder, K.; Purgahn, U.; Krautscheid, H. Selective Crystallization of Indigo B by a Modified Sublimation Method and Its Redetermined Structure. Acta Crystallogr. Sect. E Struct. Reports Online 2011, 67 (11). https://doi.org/10.1107/S1600536811040220.

(31) Salzillo, T.; D’Agostino, S.; Rivalta, A.; Giunchi, A.; Brillante, A.; Della Valle, R. G.; Bedoya-Martínez, N.; Zojer, E.; Grepioni, F.; Venuti, E. Structural, Spectroscopic, and Computational Characterization of the Concomitant Polymorphs of the Natural Semiconductor Indigo. J. Phys. Chem. C 2018, 122 (32), 18422-18431. https://doi.org/10.1021/acs.jpcc.8b03635.

(32) Susse, P.; Steins, M.; Kupcik, V. Indigo: Crystal Structure Refinement Based on Synchrotron Data. Zeitschrift für Krist. - New Cryst. Struct. 1988, 184 (3-4), 269-273. https://doi.org/10.1524/zkri.1988.184.3-4.269.

(33) Von Eller-Pandraud, H. Sur Le Polymorphisme de l'indigo. Bull.Soc.Chim.Fr. 1955, 1, 316-317.

(34) Gribova, E. A.; Zhdanov, G. S.; Golder, G. A. X-Ray Determination of the Structure of Indigo and Thoindigo. Kristallografiya 1956, 1, 53.

(35) Rivalta, A.; Giunchi, A.; Pandolfi, L.; Salzillo, T.; S. d'Agostino; Werzer, O.; Schrode, B.; Demitri, N.; MasTorrent, M.; Brillante, A.; Della Valle, R. G.; Venuti, E. Crystal Alignment of Surface Stabilized Polymorph in Thioindigo Films. Dye. Pigment. 2020, 172, 107847. https://doi.org/10.1016/j.dyepig.2019.107847.

(36) Pandolfi, L.; Rivalta, A.; Salzillo, T.; Giunchi, A.; D’Agostino, S.; Della Valle, R. G.; Brillante, A.; Venuti, E. In Search of Surface-Induced Crystal Structures: The Case of Tyrian Purple. J. Phys. Chem. C 2020, 124 (32), 17702-17710. https://doi.org/10.1021/acs.jpcc.0c05186.

(37) Salzillo, T.; Rivalta, A.; Castagnetti, N.; D’Agostino, S.; Masino, M.; Grepioni, F.; Venuti, E.; Brillante, A.; Girlando, A. Spectroscopic Identification of Quinacridone Polymorphs for Organic Electronics. CrystEngComm 2019, 21 (24), 3702-3708. https://doi.org/10.1039/C9CE00070D.

(38) Hirth, J. P.; Pound, G. M. Coefficients of Evaporation and Condensation. J. Phys. Chem. 1960, 64 (5), 
619-626. https://doi.org/10.1021/j100834a024.

(39) Macrae, C. F.; Bruno, I. J.; Chisholm, J. A.; Edgington, P. R.; McCabe, P.; Pidcock, E.; Rodriguez-Monge, L.; Taylor, R.; Van De Streek, J.; Wood, P. A. Mercury CSD 2.0 - New Features for the Visualization and Investigation of Crystal Structures. J. Appl. Crystallogr. 2008, 41 (2), 466-470. https://doi.org/10.1107/S0021889807067908.

(40) Groom, C. R.; Bruno, I. J.; Lightfoot, M. P.; Ward, S. C. The Cambridge Structural Database. Acta Crystallogr. Sect. B Struct. Sci. Cryst. Eng. Mater. 2016, 72 (2), 171-179. https://doi.org/10.1107/S2052520616003954.

(41) Ruiz-Ortega, L. I.; Schitter, G.; Mesquida, P. Electrostatic Read Out for Label-Free Assays Based on Kelvin Force Principle. Sens. Imaging 2019, 20 (1), 23. https://doi.org/10.1007/s11220-019-0244-0.

(42) Patterson, A. L. The Scherrer Formula for X-Ray Particle Size Determination. Phys. Rev. 1939, 56 (10), 978-982. https://doi.org/10.1103/PhysRev.56.978.

(43) Amar, J. G.; Family, F.; Lam, P.-M. Dynamic Scaling of the Island-Size Distribution and Percolation in a Model of Submonolayer Molecular-Beam Epitaxy. Phys. Rev. B 1994, 50 (12), 8781-8797. https://doi.org/10.1103/PhysRevB.50.8781.

(44) Liu, X.; Mohamed, S. H.; Ngaruiya, J. M.; Wuttig, M.; Michely, T. Modifying the Growth of Organic Thin Films by a Self-Assembled Monolayer. J. Appl. Phys. 2003, 93 (8), 4852-4855. https://doi.org/10.1063/1.1559935.

(45) Yoshinobu, T.; Iwamoto, A.; Sudoh, K.; Iwasaki, H. Scaling Analysis of a - and Poly -Si Surface Roughness by Atomic Force Microscopy. MRS Proc. 1994, 367 (1), 329-334. https://doi.org/10.1557/PROC-367329.

(46) Hlawacek, G.; Teichert, C. Nucleation and Growth of Thin Films of Rod-like Conjugated Molecules. J. Phys. Condens. Matter 2013, 25 (14), 143202. https://doi.org/10.1088/0953-8984/25/14/143202.

(47) Brillante, A.; Bilotti, I.; Della Valle, R. G.; Venuti, E.; Girlando, A.; Masino, M.; Liscio, F.; Milita, S.; Albonetti, C.; D’angelo, P.; Shehu, A.; Biscarini, F. Structure and Dynamics of Pentacene on SiO2: From Monolayer to Bulk Structure. Phys. Rev. B 2012, 85 (19), 195308. https://doi.org/10.1103/PhysRevB.85.195308.

(48) Pratontep, S.; Brinkmann, M.; Nüesch, F.; Zuppiroli, L. Correlated Growth in Ultrathin Pentacene Films on Silicon Oxide: Effect of Deposition Rate. Phys. Rev. B 2004, 69 (16), 165201. https://doi.org/10.1103/PhysRevB.69.165201.

(49) Hosokai, T.; Hinderhofer, A.; Bussolotti, F.; Yonezawa, K.; Lorch, C.; Vorobiev, A.; Hasegawa, Y.; Yamada, Y.; Kubozoro, Y.; Gerlach, A.; Kera, S.; Schreiber, F.; Ueno, N. Thickness and Substrate Dependent Thin Film Growth of Picene and Impact on the Electronic Structure. J. Phys. Chem. C 2015, 119 (52), 29027-29037. https://doi.org/10.1021/acs.jpcc.5b10453. 
(50) Gottardi, S.; Toccoli, T.; lannotta, S.; Bettotti, P.; Cassinese, A.; Barra, M.; Ricciotti, L.; Kubozono, Y. Optimizing Picene Molecular Assembling by Supersonic Molecular Beam Deposition. J. Phys. Chem. C 2012, 116 (46), 24503-24511. https://doi.org/10.1021/jp304561s.

(51) Kurihara, R.; Hosokai, T.; Kubozono, Y. Growth and Structure of Picene Thin Films on SiO 2. Mol. Cryst. Liq. Cryst. 2013, 580 (1), 83-87. https://doi.org/10.1080/15421406.2013.807718.

(52) Lü, B.; Elofsson, V.; Münger, E. P.; Sarakinos, K. Dynamic Competition between Island Growth and Coalescence in Metal-on-Insulator Deposition. Appl. Phys. Lett. 2014, 105 (16), 163107. https://doi.org/10.1063/1.4900575.

(53) Elofsson, V.; Lü, B.; Magnfält, D.; Münger, E. P.; Sarakinos, K. Unravelling the Physical Mechanisms That Determine Microstructural Evolution of Ultrathin Volmer-Weber Films. J. Appl. Phys. 2014, 116 (4), 044302. https://doi.org/10.1063/1.4890522.

(54) Stadlober, B.; Haas, U.; Maresch, H.; Haase, A. Growth Model of Pentacene on Inorganic and Organic Dielectrics Based on Scaling and Rate-Equation Theory. Phys. Rev. B 2006, 74 (16), 165302. https://doi.org/10.1103/PhysRevB.74.165302.

(55) Venables, J. A.; Spiller, G. D. T.; Hanbucken, M. Nucleation and Growth of Thin Films. Reports Prog. Phys. 1984, 47, 399-459.

(56) Brinkmann, M.; Graff, S.; Biscarini, F. Mechanism of Nonrandom Pattern Formation of PolarConjugated Molecules in a Partial Wetting Regime. Phys. Rev. B 2002, 66 (16), 165430. https://doi.org/10.1103/PhysRevB.66.165430.

(57) Brinkmann, M.; Biscarini, F.; Taliani, C.; Aiello, I.; Ghedini, M. Growth of Mesoscopic Correlated Droplet Patterns by High-Vacuum Sublimation. Phys. Rev. B 2000, 61 (24), R16339-R16342. https://doi.org/10.1103/PhysRevB.61.R16339.

(58) Campione, M.; Caprioli, S.; Moret, M.; Sassella, A. Homoepitaxial Growth of $\alpha$-Hexathiophene. J. Phys. Chem. C 2007, 111 (34), 12741-12746. https://doi.org/10.1021/jp068616j.

(59) Tejima, M.; Kita, K.; Kyuno, K.; Toriumi, A. Study on the Growth Mechanism of Pentacene Thin Films by the Analysis of Island Density and Island Size Distribution. Appl. Phys. Lett. 2004, 85 (17), 37463748. https://doi.org/10.1063/1.1812591.

(60) Portale, G.; Sciortino, L.; Albonetti, C.; Giannici, F.; Martorana, A.; Bras, W.; Biscarini, F.; Longo, A. Influence of Metal-Support Interaction on the Surface Structure of Gold Nanoclusters Deposited on Native SiOx/Si Substrates. Phys. Chem. Chem. Phys. 2014, 16 (14), 6649. https://doi.org/10.1039/c3cp54847c.

(61) Chiarella, F.; Perroni, C. A.; Chianese, F.; Barra, M.; De Luca, G. M.; Cataudella, V.; Cassinese, A. PostDeposition Wetting and Instabilities in Organic Thin Films by Supersonic Molecular Beam Deposition. Sci. Rep. 2018, 8 (1), 12015. https://doi.org/10.1038/s41598-018-30567-7. 
(62) Liu, C.; Khim, D.-Y.; Noh, Y.-Y. Organic Field-Effect Transistors by a Solvent Vapor Annealing Process. J. Nanosci. Nanotechnol. 2014, 14 (2), 1476-1493. https://doi.org/10.1166/jnn.2014.9101.

(63) Brinkmann, M.; Wittmann, J. C.; Chaumont, C.; André, J. J. Effects of Solvent on the Morphology and Crystalline Structure of Lithium Phthalocyanine Thin Films and Powders. Thin Solid Films 1997, 292 (1-2), 192-203. https://doi.org/10.1016/S0040-6090(96)09087-6.

(64) Ngai, J. H. L.; Leung, L. M.; So, S. K.; Lee, H. K. H. Organic Soluble Indigoids Derived from 3Hydroxybenzaldehyde for N-Type Organic Field-Effect Transistor (OFET) Applications. Org. Electron. 2016, 32, 258-266. https://doi.org/10.1016/j.orgel.2016.02.035.

(65) Nečas, D.; Klapetek, P. Gwyddion: An Open-Source Software for SPM Data Analysis. Open Phys. 2012, 10 (1). https://doi.org/10.2478/s11534-011-0096-2. 